\title{
Uso de dispositivo de control solar en aulas: impacto en la simulación dinámica de la iluminación natural
}

\author{
Use of solar control device in classrooms: impact of \\ dynamic simulation of natural lighting
}

\section{Juan Manuel Monteoliva Ayelén Villalba Andrea Elvira Pattini}

\section{Resumen}

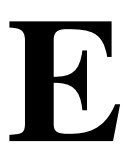

1 uso de la iluminación natural en los espacios educativos contribuye a mejorar desde diferentes perspectivas como la energía, salud y confort; sin embargo requiere de la cuidadosa planificación en su diseño. El objetivo del presente trabajo es conocer el impacto del uso de sistemas de control solar en dos tipologías de aulas (orientación N-S y E-O) representativas del parque edilicio regional, a través de herramientas predictivas dinámicas del factor iluminación natural de alta precisión. La metodología empleada fue: (a) relevamiento físico y fotométrico in situ; (b) simulación y análisis de métricas dinámicas e iluminación artificial; y (c) evaluación del impacto del uso de dispositivos de control solar (cortina textil). Los resultados evidenciaron una mayor autonomía de la iluminación natural en la orientación N-S con una alta probabilidad anual de deslumbramiento, aspecto que se revierte con el adecuado uso del dispositivo de control solar (DCS) propuesto. Asismismo, este DCS aumenta la iluminación natural disponible $(\mathrm{DAv}=12 \%)$ y disminuye su consumo de iluminación artificial, en comparación a la orientacionón (E-O). A partir de este nuevo enfoque, se busca profundizar en reducciones significativas de los consumos eléctricos (iluminación) en los edificios de uso diurno, no sólo incorporando la evaluación predictiva nuevos sistemas de iluminación artificial complementarios, sino también estrategias de control solar en pos del confort de los usuarios.

Palabras clave: Iluminación natural. Sistemas de control solar. Escuelas.

Juan Manuel Monteoliva Consejo Nacional de Investigaciones Científicas y Técnicas Mendoza - Argentina

Ayelén Villalba Consejo Nacional de Investigaciones Científicas y Técnicas Mendoza - Argentina

Andrea Elvira Pattini Consejo Nacional de Investigaciones Científicas y Técnicas Mendoza - Argentina

Recebido em 24/10/13 Aceito em 05/05/14

\section{Abstract}

The use of daylighting in educational spaces contributes from different perspectives such as energy, health and comfort. However it requires careful planning during design. The aim of this study is to determine the impact of solar control system in two classrooms (north-south orientation and east-west orientation), typical of regional educational buildings, through a dynamic daylight predictive method. The methodology adopted was: (a) physical and photometric survey in situ; (b) simulation and analysis of dynamic daylight metrics and supplemental artificial lighting; and (c) evaluation of the impact of the solar control system (textile curtain). The results indicated the empowerment of daylight in the north and south facing classroom with high annual probability of glare, something that is reversed with the proper use of the proposed solar control device $(S C D)$. Also, this $S C D$ increases daylight availability $(D A v=12 \%)$ and reduces artificial lighting consumption, compared to east-west orientation. From this new approach, further significant reductions in electricity consumption (lighting) in day-use buildings are seeked, not only by adding complementary artificial lighting systems but also by using solar control system strategies in pursuit of user comfort.

Keywords: Daylighting. Solar control system. Schools. 


\section{Introducción}

Una de las características físicas más críticas del aula es la iluminación (PHILLIPS, 1997). La importancia de la iluminación natural en los espacios educativos, entre otros, está dada por su aporte desde diferentes perspectivas como la energía, salud y confort. Esto conlleva a la necesidad de profundizar en los diseños, tecnologías y métodos de cálculos predictivos dinámicos para un mejor aprovechamiento dentro de estas instituciones. El primer paso en la evaluación de la capacidad visual y la eficiencia energética proporcionada por la luz natural requiere una estimación precisa de la cantidad de luz que ingresa a un edificio (LI; LAU; LAM, 2004). Estas decisiones afectan la cantidad y calidad de la iluminación, costos, vistas, ganancia solar y consumo energético. En ciudades donde predomina el cielo claro con sol, como el caso de Mendoza (Argentina) con un promedio anual de duración de sol de 2850 horas, la radiación solar es lo suficientemente "energética" en términos de eficacia luminosa ${ }^{1}$ (Figura 1). Esto promueve la cantidad y calidad adecuada de la luz natural en los espacios interiores para realizar tareas visuales diurnas en confort, con un ahorro energético equivalente al $50-80 \%$ del consumo energético requerido por la iluminación artificial complementaria (BODART; DE HERDE, 2002). Se denomina iluminación artificial complementaria a la iluminación artificial en edificios de uso diurno, donde ésta completa a la iluminación natural cuando no alcanza los valores mínimos de iluminancia horizontal sobre los planos de trabajo durante el período de uso del espacio. Estos valores están establecidos generalmente por las normativas vigentes de cada región, en el caso de Argentina por medio de la Norma IRAM AADL $\begin{array}{llllll}\text { J20 } & 04 & \text { (INSTITUTO..., 1974) y } & \text { J20 } & 02\end{array}$ (INSTITUTO..., 1970).

Para que esto suceda, el uso de la iluminación natural en la región como fuente dinámica iluminante, requiere de cuidadosa planificación en su diseño, principalmente en su orientación (YANNAS, 1994). Los edificos escolares en la región concentran sus actividades en los meses de temperaturas templadas y frías, con sus vacaciones anuales en los meses de verano. Si el aula es orientada al este, sólo tendra posibilidad de captación solar directa para calefacción pasiva hasta el mediodía solar (orientación oeste, después del mediodía solar) perdiendo el aporte del resto del día. Sin embargo si se orienta al norte, el asolamiento será beneficioso todo el año a partir del entendimiento y aplicación algunos principios de diseño. Este es el caso estrategias de sombreado estacional en los meses de verano (aleros) o dispositivos de control solar con capacidad de regulación (cortina textil) (BAKER; STEEMERS, 2002) que eliman el aporte de radiación directa sobre la superficie vidriada, evitando sobrecalentamiento, mientras que en los meses de invierno permiten ingresar la radiación directa necesaria para calefaccionar. De no ser así, la luz solar disponible, será eliminada por falta de confort térmico-lumínico. Es decir, se anulará el aporte de luz natural y generará espacios sombríos (perdiendo la disponibilidad de luz natural característica de la región); consumiendo así mayor energía eléctrica en iluminación artificial complementaria (PATTINI, 2009).

\section{Figura 1 - Disponibilidad de Radiación Solar Directa (RSD) anual para Mendoza, Argentina}

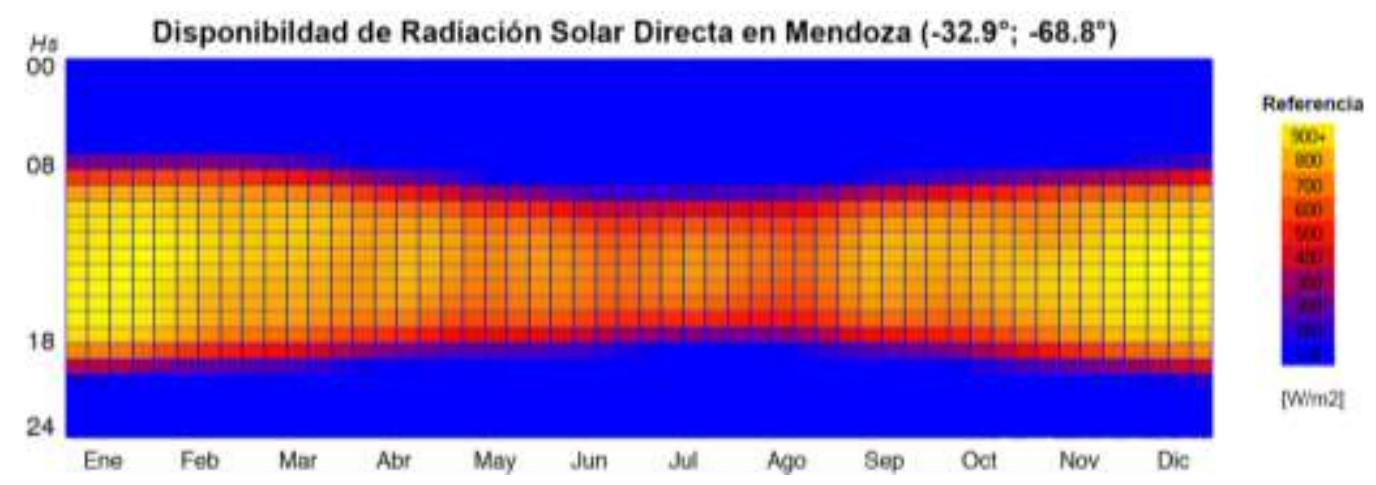

${ }^{1}$ Eficacia luminosa: cociente entre la iluminancia e irradiancia y sus valores dependerán de la altitud del sol, de la nubosidad y de la concentración de aerosoles y vapor de agua en la atmósfera 
La última década ha sido testigo de múltiples avances en la forma de analizar numéricamente el comportamiento general de la iluminación natural en los diversos espacios. Entre los principales avances se puede mencionar la aparición de un nuevo paradigma dinámico. Éste incorpora el uso de archivos climáticos y distribución de luminancia de cielo (PÉREZ; SEALS; MICHALSKY, 1993; REINHART; MARDALJEVIC; ROGERS, 2006) generando un análisis predictivos anual de mayor precisión del factor iluminación natural. El entorno de simulación empleado por este nuevo paradigma es DAYSIM. DAYSIM (REINHART et al., 2011) es una herramienta basada en RADIANCE (WARD; SHAKESPEARE, 1998) y emplea métodos de optimización del cálculo de iluminancia y distribución de luminancia bajo distintas condiciones climáticas - información climática del sitio en estudio - a lo largo del año, bajo el Modelo Pérez de Cielo (PEREZ et al., 1990; PEREZ; SEALS; MICHALSKY, 1993). Asimismo aporta diferentes métricas dinámicas para el análisis predictivo del factor de iluminación natural como: DF (coeficiente de luz diurna), DA (luz natural autónoma), DAcon (iluminación natural autónoma ponderada), DAv (iluminación natural disponible) entre otras. La justificación del uso de DAYSIM como entorno de simulación, se debe a limitaciones encontradas en software similares como EnergyPlus (VERSAGE; MELO; LAMBERTS, 2010; RAMOS; GHISI, 2010), que demuestran que el algoritmo empleado por esta herramienta sobreestima los valores de iluminancia para los puntos más lejanos a los aventanamientos. Sin embargo, como es reconocido el uso masivo de esta herramienta en el análisis energético predictivo, los output brindados por DAYSIM (consumos energéticos de iluminación artificial complementaria) son totalmente compatibles y exportables a EnergyPlus, como a otros software de similares características Esp-R, TRNSYS.

A partir de lo expuesto, el siguiente trabajo tiene por objetivo conocer el impacto del uso de dispositivos de control solar (cortinas textil) en dos tipologías características de aulas a través de herramientas predictivas dinámicas del factor iluminación natural de alta precisión. Esto permitirá cuantificar el impacto del uso de sistemas de control solar en el consumo energético (iluminación artificial complementaria) y estimar el confort visual de los usuarios en los espacios analizados. La importancia de combinar el uso de espacios reales con modelos predictivos de precisión - simulaciones - radica en un mejor entendimiento de la problemática abordada. Es decir, a partir de las referencias otorgadas por mediciones y relevamientos in situ de los espacios reales, es posible ingresar y calibrar con mayor precisión los input de los simuladores. En el caso de las métricas dinámicas estos input corresponden especificamente a la geometría del espacio, materiales y bases climáticas (MONTEOLIVA; VILLALBA; PATTINI, 2012). Asimismo, de cumplirse con el adecuado uso de los mismos, estas herramientas predictivas otorgan una mayor representatividad del comportamiento de la iluminación natural en el interior de un espacio determinado. De nor ser así, la evaluación predictiva generaría datos no representativos e interpretaciones erróneas para posibles intervención en el diseño de estrategias de iluminación natural. Cabe destacar que este trabajo forma parte de una tesis doctoral en proceso denominada: "Iluminación en aulas. La dinámica de la luz natural en cielos claros y su incidencia en el rendimiento atencional de los alumnos." (MONTEOLIVA, 2010).

Uno de los ámbitos en los que la mayoría de los niños juega, aprende, transita e interactúa gran parte de su jornada diaria es el "educativo". La iluminación natural en edificios escolares es una de las características físicas más influyentes del aula (PHILLIPS, 1997) y debe ser considerada una variable del ambiente educativo a estudiar en profundidad (DUNN et al., 1985). No sólo, por la oportunidad de ahorros energéticos-ambientales (ya que de la distribución de los consumos eléctricos en edificios escolares, el $66 \%$ del gasto eléctrico se realiza en las aulas) que ésta representa, sino también por la importancia de su ambiente visual. Éste incide en la capacidad de un estudiante para percibir estímulos visuales afectando su actitud mental (TAYLOR; GOUSIE, 1988; EVANS; FERGUSON, 2011) y por lo tanto su rendimiento (HESCHONG, 1999; OKURA; HESCHONG; WRIGHT, 2000; WU; NG, 2003). Es posible resaltar entonces la importancia de la iluminación natural en los espacios educativos desde diferentes perspectivas como la energía, salud y confort. Esto genera la necesidad de profundizar en los diseños y tecnologías y métodos de cálculos predictivos dinámicos, para un mejor aprovechamiento de la luz natural dentro de estas instituciones (MONTEOLIVA; PATTINI, 2013).

\section{Caso de estudio}

El criterio empleado para la selección del caso de estudio fue edificios educativos públicos con tipologías de aulas características de la región. La institución seleccionada fue la Escuela República de Chile $\mathrm{N}^{\mathrm{o}}$ 1256. Este edificio se encuentra emplazado a $3 \mathrm{~km}$ de la capital, provincia de Mendoza (32.52.49 S, 68.52.45 E -853msm-); sitio 
de densidad edilicia media con obstrucciones parciales a los aventanamientos generadas por edificios cercanos y arbolado (Figura 2). La arquitectura general presenta una tipología en bloque de aulas compacto con desarrollo principal este (E). Cada ala (norte y sur), simétricas entre sí, posee seis (6) aulas - cuatro (4) con orientación este (E) y dos con orientación norte (N) -, vinculadas por un pasillo de circulación cubierto. Dentro de la institución fueron escogidas dos tipología de aulas diferentes denominadas (A2) y (A3).

\section{Metodología}

La metodología empleada fue dividida en tres etapas principales:

(a) relevamiento físico y fotométrico de tipologías de aulas reales (sistemas de iluminación natural);

(b) simulación y análisis de métricas dinámicas e iluminación artificial complementaria; $y$

(c) uso de dispositivos de control solar.

Análisis del impacto en las autonomías de los espacios y sus consumos eléctricos en iluminación artificial complementaria.

\section{Relevamiento físico y fotométrico de tipologías de aulas seleccionadas}

Las aulas seleccionadas (A2) y (A3), presentan tipología de bloque compacto de $7.95 \mathrm{mts} \times \mathrm{mts} \times$ $3.86 \mathrm{mts}\left(47.7 \mathrm{~m}^{2}\right)$ con un sistema de aventanamiento bilateral, sin dispositivos de control solar. El sistema de iluminación artificial complementario instalado es de 6 artefactos -2 tubos fluorescentes T8 de 36 [W] cada uno de $4300^{\circ} \mathrm{K}$ (luz neutra), sin pantalla difusora-. Asimismo presentan las siguientes cualidades distintivas:

(a) Aula 2 (A2): aula con orientación (E-O). La principal superficie vidriada se ubica en la fachada
(E), a la altura de visión, abarcando aproximadamente un $90 \%$ del largo la misma. Sin embargo el acceso de la radiación solar actualmente se ve obstaculizado parcialmente por arbolado exterior de especie plátano. Una de las tipología más representativa del arbolado urbano de la ciudad de Mendoza (CANTÓN; DE ROSA; KASPERIDUS, 2003). Si bien, la mayoría de especies presentes en modelo oasis son del tipo caducifolio, el plátano es de follaje perenne, con la particularidad de mantener la hoja durante todo el año, a partir de su condición de marcescente (CÓRICA, 2009). La relación entre la superficie vidriada (SV) y superficie de piso (SP) (Norma IRAM AADL J20 04(INSTITUTO..., 1974)) es del 13\%; y

(b) Aula 3 (A3): aula con orientación (N-S). El sistema de iluminación natural consta principalmente de una superficie vidriada ubicada en la fachada (S), a la altura de visión, abarcando aproximadamente un $90 \%$ del largo de la misma. En relación a los aventanamientos de la fachada $(\mathrm{N})$, se ubica en la parte superior y se encuentra parcialmente obstaculizado por arbolado exterior de especie ciprés. Como toda conífera, son de hojas perenne y presentan un porte piramidal. La relación entre (SV) y (SP) es del 19\%.

En cuanto a las características fotométricas, y específicamente la información concerniente a las propiedades ópticas de las superficies, fueron obtenida a partir de mediciones in situ. Se determinaron las luminancias de los materiales predominantes en el ambiente (cielorraso, muros y solados) para caracterizarlos a partir de sus reflectancias en el modelo virtual. Estas mediciones se llevaron acabo de acuerdo al protocolo de medición de Fontoynont (1999). El instrumental empleado fue un luminancímetro Minolta LS 110 (certificado de calibración, ángulo de lectura de $1 / 3^{\circ}$ y rango de medición de 0.01 a $999.900 \mathrm{~cd} / \mathrm{m} 2$ ) y cartillas patrón Kodak.

Figura 2 - Escuela República de Chile No1256 (Mendoza, Argentina 32.52.49 S, 68.52.45 E)
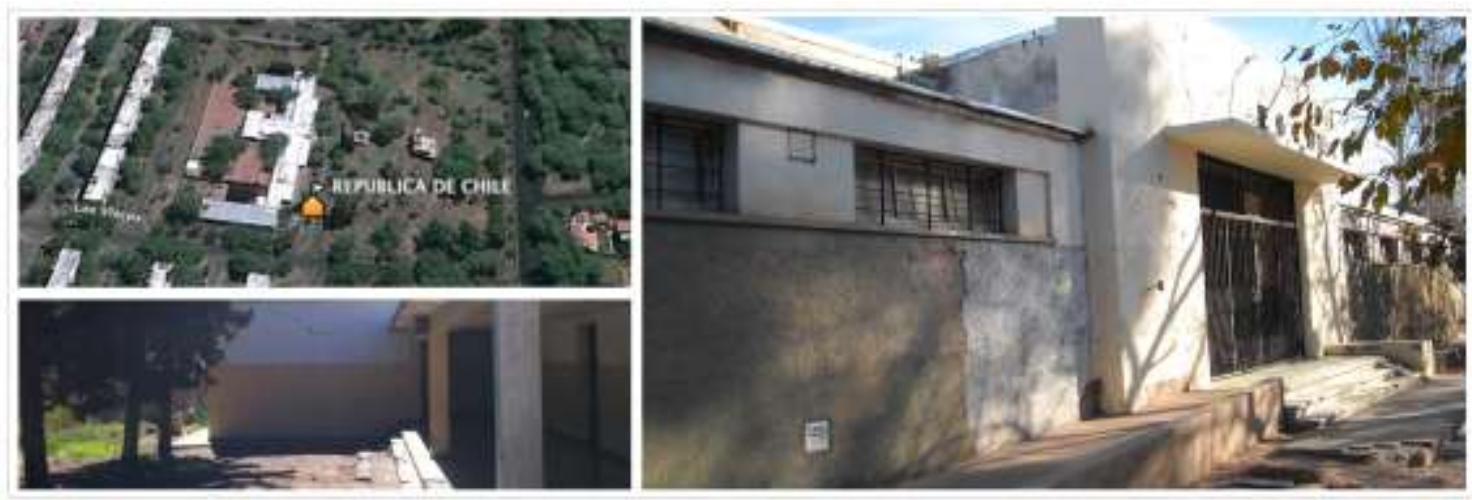

46 Monteoliva, J. M.; Villalba, A.; Pattini, A. E. 


\section{Simulación y análisis de métricas dinámicas e iluminación artificial complementaria}

Como fue mencionado previamente, a partir de las mediciones y relvamientos realizados in situ, fue calibrada la herramienta de simulación. A continuación se describen algunos de los aspectos metodológicos más importantes.

\section{Configuración del entorno}

Para poder generar la simulación de métricas dinámicas, fue necesario en primera instancia, establecer:

(a) el valor mínimo de iluminancia horizontal requerido en el espacio; y

(b) la cantidad y distribución de los sensores de medición o adquisidores de datos. Los parámetros fueron:

- valor mínimo de iluminancia horizontal sobre puesto de trabajo de 500 [lux] a partir del criterio empleado en la normativa nacional vigente para edificios escolares (Norma IRAM ${ }^{2}$ AADL $^{3}$ J20 04(INSTITUTO..., 1974)) y los Criterios y Normativa Básica de Arquitectura Escolar (MINISTERIO..., 1998); y

- grilla de medición de iluminancia de veinte (20) puntos por aula.

La grilla fue generada a partir del plano útil a $80 \mathrm{~cm}$ sobre el solado del local - considerado como la zona del plano de trabajo, - determinada por las líneas trazadas a $1 \mathrm{mt}$. de las paredes -

(INSTITUTO..., 1970). Debido al análisis propuesto de precisión, fue duplicada la cantidad de puntos requeridos por Protocolo de medición del factor iluminación en puestos de trabajo de la Superintendencia de Riesgos de Trabajo (SRT) (Eq. 1). Toda la normativa de aplicación general de la SRT, puede ser consultada en la sección de Información Legislativa del Centro de Documentación e Información del Ministerio de Economía y Finanzas Públicas: InfoLEG (www.infoleg.gov.ar).

Puntos del Grillado $=\left(\frac{A \times L}{\operatorname{hm} \times(A \times L)}+2\right)^{2}$

Eq. 1

Dnde:

$A$ es el ancho;

$L$ el largo; y $\mathrm{hm}$ altura de montaje, siendo $(\mathrm{hm})$ la distancia vertical considerada entre la superficie de trabajo y la fuente artificial.

\section{Parámetros de simulación}

Los parámetros establecidos corresponden a una escena simple con elementos básicos translúcidos, transparentes y opacos, sin ningún sistema de iluminación natural complejo (REINHART, 2006) y éstos son:

(ab) inter-reflexiones 5;

(ad) divisiones 1000;

(as) muestreo 20;

(aa) precisión 0.1 ;

(ar) resolución 300;

(dt) umbral directo $0 ; \mathrm{y}$

(ds) sub-muestreo directo 0 .

Éstos fueron desarrollados en su totalidad bajo el entorno RADIANCE (WARD; SHAKESPEARE, 1998) y DAYSIM (REINHART et al., 2011) integrados en la herramienta DIVA - generada para el entorno de Rhinoceros 3D (MCNELL et al., 2010). DIVA es una herramienta de análisis predictivo dinámico del factor de iluminación natural y consumo energético (JACUBIEK; REINHART, 2011). Esta herramienta emplea métodos de optimización del cálculo de iluminancia y distribución de luminancia bajo distintas condiciones climáticas -información climática del sitio en estudio - a lo largo del año, bajo el Modelo Pérez de Cielo (PEREZ et al., 1990; PEREZ; SEALS; MICHALSKY, 1993). El paquete climático utilizado fue Mendoza_MZ, adquirido recientemente por el Instituto de Ciencias Humanas, Sociales y Ambientales (INCIHUSA CCT CONICET Mendoza).

Asimismo, fue generado un archivo de ocupación del espacio a partir de los establecido por la Dirección General de Escuelas (DIRECCIÓN..., 2012). Sobre la base de la información proporcionada de los días y horas de uso del espacio educativo (lunes a viernes de 8:00 a 18:00 con vacaciones invernales-estivales y feriados nacionales), fue generado un perfil anual de ocupación (REINHART et al., 2011) (Figura 3). El criterio de no incorporar al estudio los períodos de recesos (vacaciones estivales e invernales) se debe que, a diferencia de otros países, durante éstos no se realizan actividades extra-curriculares (MONTEOLIVA; PATTINI, 2013).

2Instituto Argentino de Normalización y Certificación (IRAM).

3Asociación Argentina de Luminotecnia (ADDL). 
Figura 3 - Archivo de ocupación del espacio generado (escuelaconvacaciones.csv)

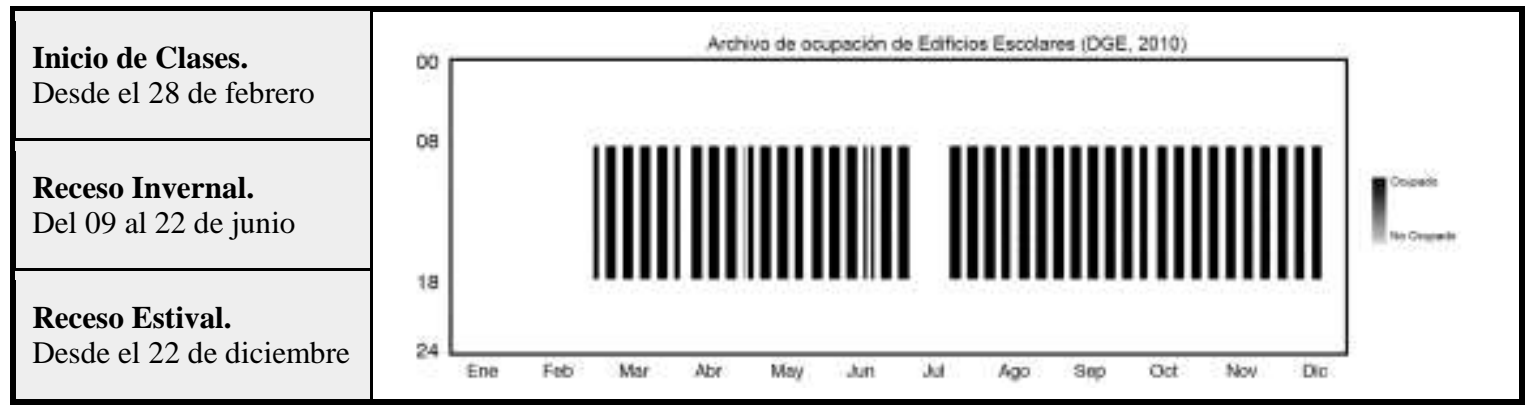

Dada la característica de cielo claro de la región y los objetivos propuestos, las métricas dinámicas estudiadas fueron: luz natural autónoma (DA500lux) daylight autonomy, luz natural autónoma ponderada (DAcon500lux) continouos daylight autonomy y luz natural disponible daylight availibility. Se denomina luz natural autónoma de 500 [lux] (DA500lux), al porcentaje de tiempo durante el cual se mantiene el nivel mínimo de 500 [lux] sobre el plano de trabajo; mientras que la luz autónoma ponderada (DAcon500lux) (ROGERS, 2006) otorga créditos parciales a los valores cercanos al valor de consigna requerido de 500 [lux]. Por otro lado, la luz natural disponible daylight availibility (DAv), establece un intervalo producido por el valor mínimo requerido por la luz autónoma (DA500lux) y 10 veces este valor (en este caso, valores hasta 5000 [lux]), exceso de iluminación natural que supone al menos el 5\% del año de trabajo. Asimismo esta métrica puede ser profundizada a partir de otro indicador como la iluminación natural útil o useful daylight index (UDI), específicamente su rango de valores superiores a 2000 [lux] (UDI200lux) para predecir posibles situaciones de deslumbramiento o falta de confort de los enocupantes de un espacio. Sin embargo, en este estudio, esta estimación, fue profundizada a partir del análisis del deslumbramiento anual $(D G P)$. La respuesta a tu pregunta es que las áreas en rojo son las que reciben 10 veces la iluminancia umbral establecido durante más de $5 \%$ del tiempo ocupado durante el año.

Posteriormente el análisis fue complementado con el estudio del área iluminada "daylit area", la probabilidad de deslumbramiento anual "annual glare" y el porcentaje de horas de uso de dispositivos del control solar (Uso DCS). El indicador área iluminada, establece un cierto umbral de iluminancia (DA500lux) que tiene que ser logrado al menos el $50 \%$ de las horas ocupadas, considerando este espacio acondicionado con iluminación natural. Este indicador reporta el porcentaje de área de piso que es iluminado por luz natural. El procedimiento tiene presenta la ventaja de brindar un porcentaje unficado de la grilla, o bien valores puntual para el análisis de zonas de bajo rendimiento.

\section{Sistemas de iluminación artificial complementaria}

El sistema propuesto y evaluado fue de fotosensor con dimerizado (Fd) para las tipologías (A2) y (A3) en condiciones sin dispositivos de control solar (Sin DCS) y con dispositivos de control solar (Con DCS). La justificación del empleo de esta tecnología está basada en dos aspectos:

(a) limitaciones en el algoritmo Lightswitch (REINHART, 2004) para modificar el valor mínimo de iluminación establecido por defecto en 250 [lux] - siendo la mitad a lo requerido como mínimo en estos espacios; y

(b) la necesidad de buscar nuevas soluciones tecnológicas a la problemática de los acondicionamientos lumínicos en las aula.

Los parámetros empleados en $(\mathrm{Fd})$ : potencia total de los artefactos 500 [W], iluminancia requerida 500 [lux], factor de pérdida en el balasto $12 \%$ y cantidad de energía perdida durante período de apagado - especial en sistemas automáticos- 15.0 valores por defecto. La potencia total propuesta de 500 [W], se debe en analogía a la situación típica de espacios educativos de 6 artefactos de 2 tubos fluorescentes T8 de 36 [W] cada uno - sin pantalla difusora.

\section{Uso de dispositivos de control solar: análisis del impacto en las autonomías de los espacios y sus consumos eléctricos en iluminación artificial complementaria}

A través de DAYSIM, DIVA, permite el modelado de espacios con múltiples dispositivos de control solar (persianas, cortinas y vidrios electrocrómicos). El procesamiento realizado por DAYSIM genera automáticamente varios perfiles 
de iluminancia anual correspondientes a los diferentes estados (por defecto abierto-cerrado) de los dispositivos de control solar (DCS). En una etapa post-procesamiento, emplea el modelo Lightwitch (REINHART, 2004). Este algoritmo integrado a DIVA predice, a partir del criterio anual de ocupación del espacio propuesto (escuelaconvacaciones.csv) y la iluminancia mínima requerida sobre plano de trabajo (500 [lux]), el porcentaje de horas de uso de los dispositivos de control solar, como así también, el consumo energético anual [KWh] del sistema de iluminación artificial complementario propuesto $(F d)$.

En ventanas de envolventes, particularmente con acceso a radiación solar, es habitual la presencia de elementos de control solar que permitan regular el ingreso de la radiación solar visible. Uno de los elementos más empleados con este fin son las cortinas textiles. Éstas presentan la característica de ser sistemas de control solar de transmitancia homogénea, confeccionadas en materiales opacos o difusores y ubicadas en la parte interior de los sistemas de aventanamiento. Asimismo pertenecen a la categoría de pantallas flexibles, respondiendo a su capacidad de regulación (BAKER; STEEMERS, 2002), siendo de enrollar o de correr verticalmente. Esto favorece la regulación tanto de la vista al exterior (privacidad) como de la radiación incidente en zonas interiores cercanas a la ventana.

A partir de lo mencionado, fue seleccionado como sistema de control solar para su estudio, la cortina textil interior confeccionada a partir del género tropical (nombre vulgar). La selección de este género, está basada en ser uno de los tres textiles de uso más frecuente en edificios no residenciales de la ciudad oasis de Mendoza (VILLALBA; PATTINI; CÓRICA, 2012); y específicamente ser éste, el más empleado en el ámbito educativo regional. Este textil forma parte de los denominados "tejidos cerrados" $(0 \%$ a $7 \%$ de apertura) de "hilados de color intermedio" con una reflectancia del $42 \%$ y un índice de transmitancia visible (TV) de 0.24. (Tabla 1) (VILLALBA; MONTEOLIVA; PATTINI, 2012).

A partir del dispositivo de control solar mencionado, se genera un análisis de su impacto en los espacios (A2) y (A3) y su relación con las métricas dinámicas seleccionadas (DA500lux, DAcon500lux y DAv), el porcentaje del área iluminada y consumos eléctricos anuales por iluminación artificial complementaria [KWh].

\section{Resultados}

\section{Relevamiento físico y fotométrico de tipologías de aulas reales}

En el siguiente apartado se presentan el relevamiento físico de las tipologías de aulas analizadas en el caso de estudio (Figura 4) y las propiedades fotométricas de materiales relevados in situ (Tabla 2).

\section{Simulación y análisis de métricas dinámicas e iluminación artificial complementaria}

En el siguiente apartado se analizan los espacios seleccionados (A2) y (A3) sin dispositivos de control solar (Sin DCS). Es decir, en esta primera instancia se reproducen las condiciones del relevamiento in situ, donde los espacios no estaban acondicionados con sistemas de control solar. La métricas dinámicas evaluadas son: luz natural autónoma (DA500lux), luz natural autónoma ponderada (DAcon500lux), luz natural disponible $(D A v)$, área iluminada, deslumbramiento anual (DGP) y consumos eléctricos del sistema de iluminación artificial complementario $(F d)$.

Tabla 1 - Caracterización del dispositivo de control solar, textil tropical beige

\begin{tabular}{llll}
\hline \multicolumn{2}{c}{ Vista del Material } & \multicolumn{2}{c}{ Características del Dispositivo de Control Solar (DC) seleccionado } \\
\hline & & Textil & Tropical beige \\
& & Color & beige \\
& & Composición & $100 \%$ poliéster \\
& & Factor de apertura & $7 \%$ \\
& & Conductividad & $0.17 \mathrm{~W} / \mathrm{m} 2 \mathrm{~K}$ \\
& & Espesor & $0.5 \mathrm{~mm}$ \\
& & Albedo & $45 \%$ \\
\hline & & Transmitancia solar (TS) & $20 \%$ \\
& & Transmitancia visible (TV) & $24 \%$ \\
\hline
\end{tabular}


Figura 4 - Caracterización de los casos de estudio relevados y reproducidos en CAD

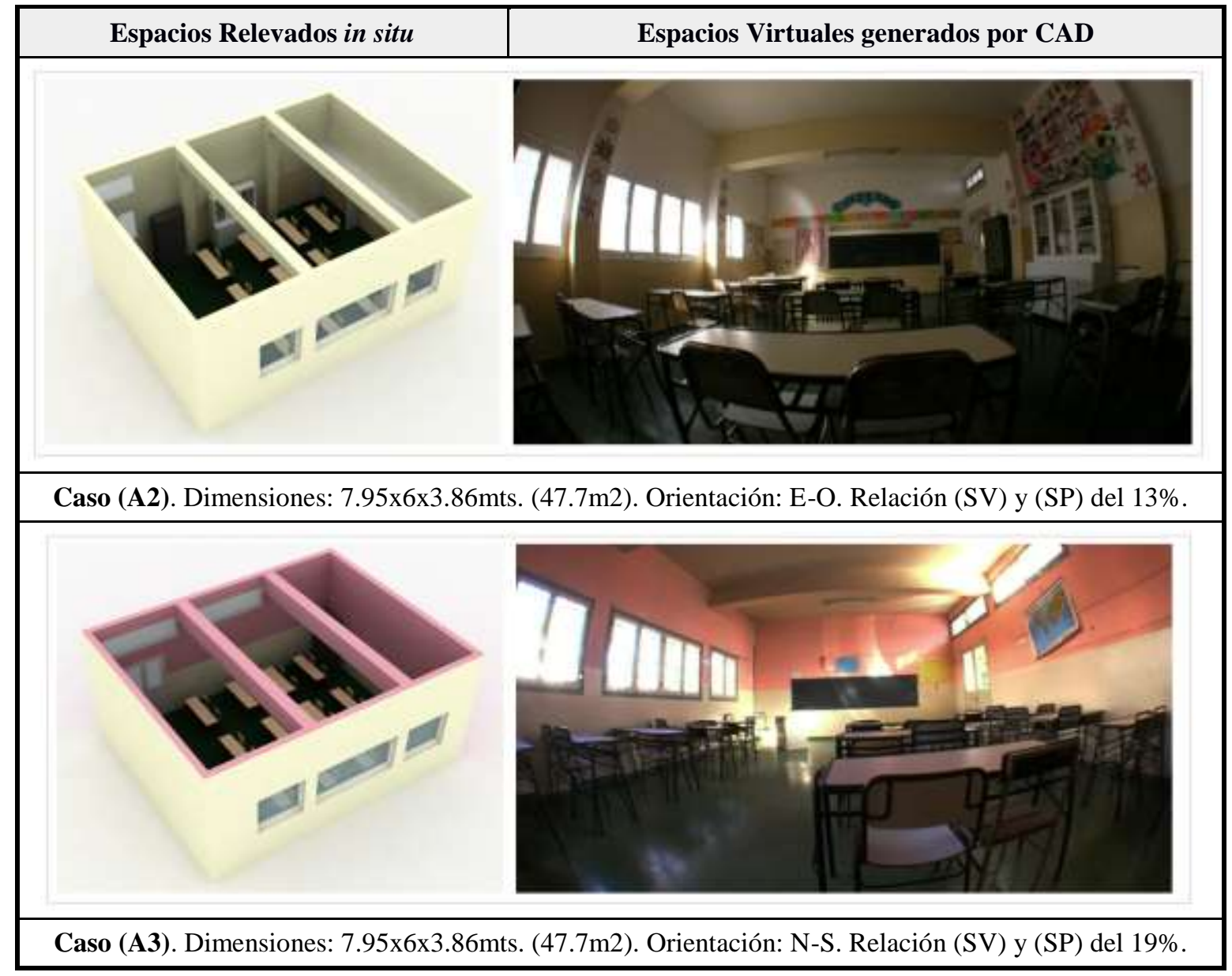

Tabla 2 - Propiedad ópticas de los materiales y características del grillado en el plano útil

\begin{tabular}{lll}
\hline & Factor de Reflectancia [\%]* & Materiales RADIANCE \\
\hline Cielorraso & $70-80[\%]$ & beige paint LBNL 2k216 \\
Muros & $40-60[\%]$ & beige 2k210 LES091 \\
Solados & $10-30[\%]$ & beige-brown 3k313 LES091 \\
Vidrio & T $0.91 \mathrm{Ra} 0.082$ Rp 0.082 (Pilkington North & CLEAR2 LO \\
& America.) & \\
\hline
\end{tabular}

Nota: *Los factores de reflexión recomendados para las superficies interiores de las aulas por la normativa nacional vigente IRAMM AADL J20 04 y CyNBAE (1998) coincidentes con los factores relevados in situ.

Los resultados en las métricas dinámicas, muestran un mejor acondicionamiento en el espacio $(A 3)$ en relación a (A2). Esto puede observarse a través de la métrica luz natural autónoma (DA500lux) con variaciones (DDA500lux) del $23 \%$, como así también en la luz natural ponderada (DAcon500lux) con variaciones ( $\triangle D A$ con500lux) del $13 \%$. Sin embargo, estas métricas analizan tan sólo el porcentaje de tiempo durante el cual se mantiene el nivel mínimo de 500 [lux], o bien cercanos al valor - ponderado - sobre el plano de trabajo. Por esta razón es incorporado al análisis, la métrica iluminación natural disponible $(D A v)$. Ésta permite profundizar en el análisis anual de los rangos útiles de iluminancias horizontales del espacio conjugando no sólo los valores mínimos establecidos como criterio (DA500lux), sino también valores no superiores a 2000 [lux] (valores generadores de posibles situación de insatisfacción o falta de confort). La diferencia en la iluminación natural disponible $(\triangle D A v)$ entre (A3) y (A2) fue del $28.2 \%$, mientras que en el área iluminada ( $\triangle$ Daylit) fue del $55 \%$ (Tabla 3 ). Por otra parte, los consumos eléctricos del sistema de iluminación artificial complementario de fotosensor y dimerizado $(F d)$ (FiguraS 5 y 6 )

50 Monteoliva, J. M.; Villalba, A.; Pattini, A. E. 
fueron para (A2) de 373.1 [KWh] y (A3) de 306.5 [KWh]. La diferencia predicha entre ambos es de 66.6 [Kwh].

En una etapa previa a la incorporación de dispositivos de control solar, fue necesario conocer el comportamiento de la fuente natural "potencialmente" deslumbrante en estos espacio y posibles perturbaciones producidas en sus usuarios. La herramienta empleada fue la evaluación de deslumbramiento anual (DGP) (FiguraS 7 y 8). Ésta presenta un nuevo paradigma dinámico, en relación a los método estático existente hasta hace una década, denominado point-in-time (situaciones "estáticas" en un determinado momento en el tiempo). Esta evaluación (DGP) emplea modelos virtuales en entorno de simulación RADIANCE-DAYSIM (WARD; SHAKESPEARE, 1998), evaluación del indicador

DGP

(WIENOLD;
CHRISTOFFERSEN, 2005) - probabilidad de deslumbramiento por fuente natural - y evaluaciones subjetivas de los usuarios - validado por Wienold y Christoffersen (2006). Se basa en la herramienta evalglare (WIENOLD, 2004), la cual a partir de la predicción por cada hora de luz natural disponible del año, genera imágenes semiesféricas $\left(180^{\circ}\right)$ por medio del motor de simulación RADIANCE. Todas estas imágenes son analizadas posteriormente por evalglare que calcula la probabilidad de que una persona se vea perturbada por deslumbramiento (DGP). Los resultados se basan en evaluaciones generadas en usuarios, a partir de escalas de calificación del deslumbramiento: imperceptible $\quad \mathrm{DGP} \geq .45$, perceptible $\quad .45>\mathrm{DGP} \geq .4, \quad$ perturbador $.4>\mathrm{DGP} \geq .35$ e intolerable $.35>$ DGP. Los resultados obtenidos para los espacios seleccionados son graficados en las Figura 7 y 8.

Tabla 3 - Resultados obtenidos de las métricas dinámicas y consumos eléctricos del sistema de iluminación artificial complementaria en los espacios "sin dispositivos de control solar" (Sin DCS)

\begin{tabular}{lcccccccc}
\hline & \multicolumn{9}{c}{ Métricas Dinámicas [\%] } & & Parámetros \\
& & $\begin{array}{c}\text { DA500lu } \\
\mathbf{x}\end{array}$ & $\begin{array}{c}\text { DAcon500lu } \\
\mathbf{x}\end{array}$ & DAv & $\begin{array}{c}\text { Área } \\
\text { Iluminada }\end{array}$ & DGP & $\begin{array}{c}\text { Consumo } \\
{[\text { [KWh] }}\end{array}$ & RADIANCE \\
\hline A2 & Sin DCS & 34 & 62 & 14,1 & 25 & 2 & 373,1 & \multirow{2}{*}{ ab2 -ad1000 } \\
\hline A3 & Sin DCS & 57 & 75 & 42,3 & 80 & 48 & 306,5 & \\
\hline
\end{tabular}

Figura 5 - Uso predictivo del sistema de iluminación artificial (Fd) en el espacio (A2)

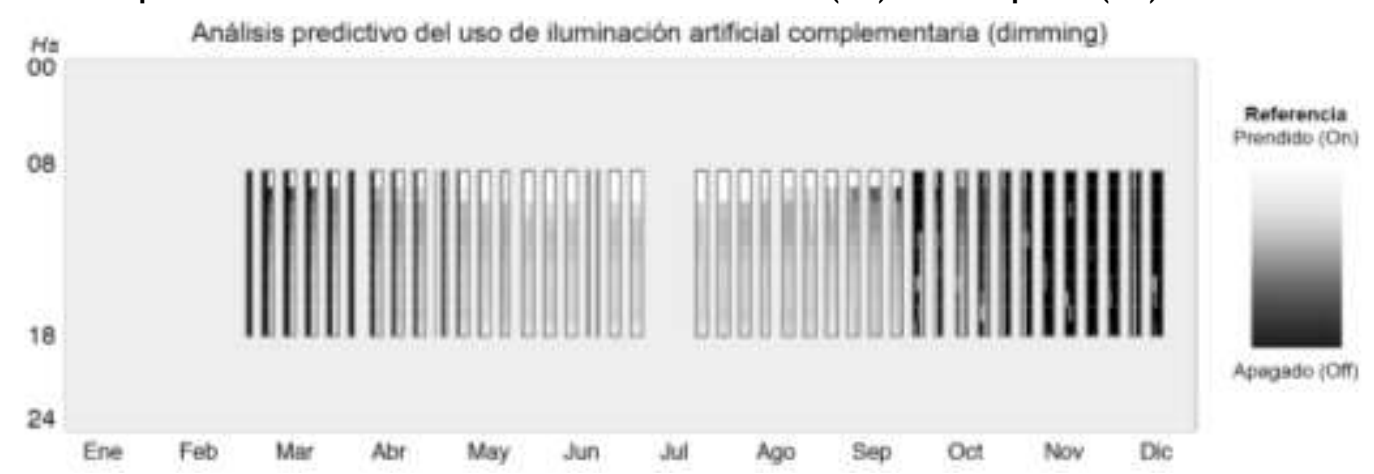

Figura 6 - Uso predictivo del sistema de iluminación artificial (Fd) en el espacio (A3)

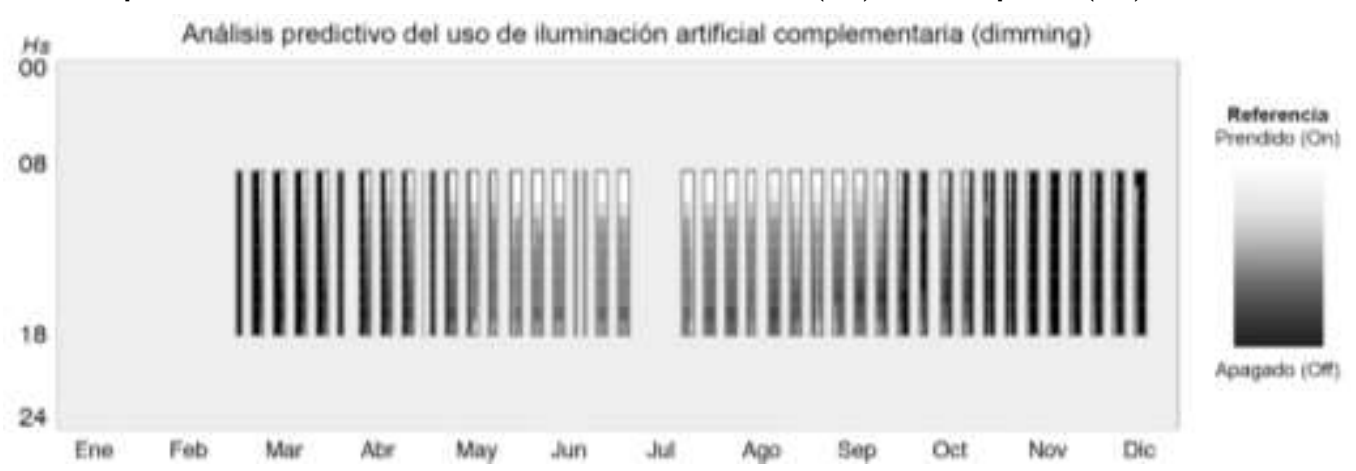


Figura 7 - Análisis de deslumbramiento anual sin dispositivos de control solar del espacios (A2)

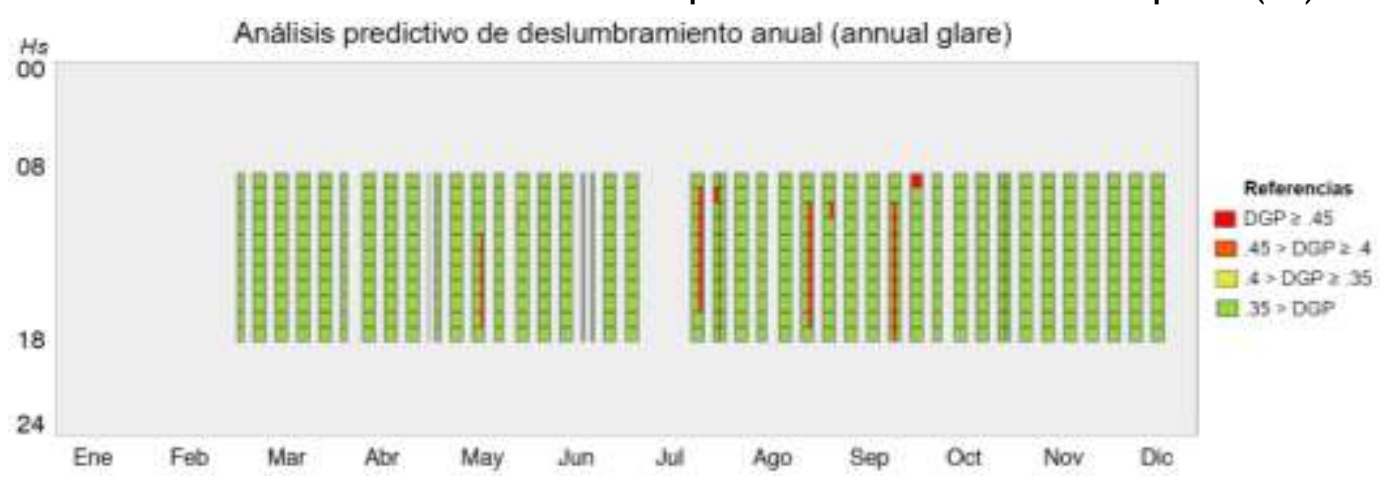

Figura 8 - Análisis de deslumbramiento anual sin dispositivos de control solar del espacios (A3)

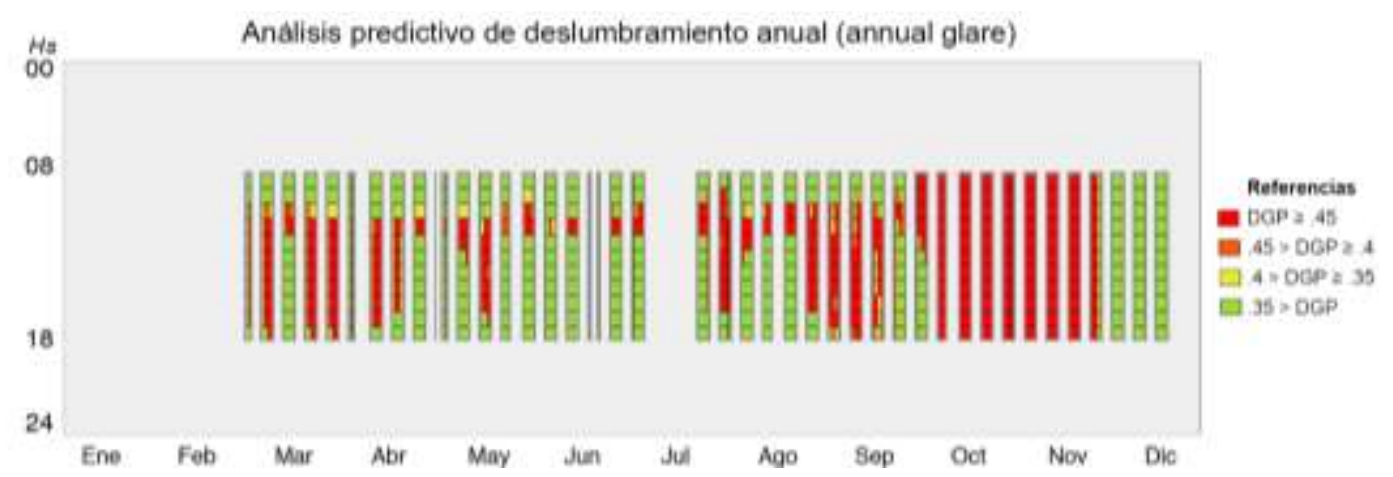

Como se puede observar en la Figura 7 y 8 , se encuentra una diferencia significativa entre los porcentaje obtenidos en la predicción del deslumbramiento anual de (A2) y (A3). En el caso (A3), el porcentaje anual de posibles situaciones de deslumbramiento intolerable (.35>DGP) alcanzan el $48 \%$, mientras que en ( $A 2)$, esta probabilidad, se reduce al $2 \%$. Cabe destacar que el porcentaje de (DGP) obtenido en (A2), está influenciado por el sombreado forestal. De no ser así, el porcentaje sería mucho mayor, ya que esta orientación recibe incidencia de rayos muy bajos (principalmente durante el invierno) que penetran directamente en el interior del espacio a las primeras horas de la mañana. Por otro lado, como era de esperar en la orientación norte $(A 3)$, si bien presenta mayor exposición a la radiación directa - mayor disponibilidad del recurso -, también presenta mayor criticidad en relación a posibles situaciones de falta de confort o deslumbramiento durante la jornada.

Para profundizar en el adecuado acondicionamiento lumínico en aulas, se incorpora en el siguiente apartado un dispositivo de control solar a los espacios estudiados (A2) y (A3). El objetivo es conocer la incidencia del uso este dispositivos de control solar - como estrategia de control de la iluminación natural - y su impacto en las autonomía de la iluminación natural y los consumos eléctricos de iluminación artificial complementaria $(F d)$.

\section{Uso de dispositivos de control solar: análisis del impacto en las autonomías de los espacios y sus consumos eléctricos en iluminación artificial complementaria}

El Dispositivo de Control Solar (DCS) seleccionado y empleado en este trabajo fue una cortina textil interior denominada tropical nombre vulgar - (Figura 9). Este material fue incorporado a los materiales por defecto de RADIANCE a partir de los parámetros establecidos en la Figura 9. Cabe destacar que los aventanamientos intervenidos con (DCS) fueron en (A2) el aventanamiento ubicado en la fachada (E) y en (A3) el aventanamiento superior en la fachada (N).

A continuación se analizan los espacios (A2) y (A3) "con dispositivos de control solar" (Con $D C S)$. La métricas dinámicas evaluadas en esta nueva instancia fueron: luz natural autónoma (DA500lux), luz natural autónoma ponderada (DAcon500lux), luz natural disponible ( $D A v)$, área iluminada y consumos eléctricos del sistema de iluminación artificial complementario $(F d)$ (Tabla 4).

52 Monteoliva, J. M.; Villalba, A.; Pattini, A. E. 
Los resultados obtenidos en las métricas dinámicas "con dispositivos de control solar" (Con DCS) marcaron, como era de esperar una reducción en las autonomía (DA500lux) y autonomías ponderadas (DAcon500lux) de la iluminación natural. En (A2) la reducción fue del $21 \%$ para ambas métricas; mientras que en (A3) fue del $3 \%$ y $1 \%$, respectivamente. Esto permite inferir que el espacio (A2) presenta niveles elevados durante importantes períodos del año >2000 [lux]. Sin embargo, estos valores no son lo suficientemente "intensos" para que al ser interceptados por un dispositivo de control solar alcance los valores establecidos como criterio mínimo de 500 [lux]. Es decir, dada la orientación este (E) del espacio, las intensidades durante las mañanas son altas (>2000 [lux]), sin embargo durante el resto de la jornada los valores promedios predominantes son $<500$ [lux]. Por otro lado, en el caso (A3) se observa una reducción mínima en (DA500lux) y (DAcon500lux) del $2 \%$ promedio. Esto nos permite conocer la alta disponibilidad del recurso con el que cuenta la orientación norte. Es decir, es tan alta la disponibilidad de radiación solar directa con valores >2000 [lux], que al ser interceptada por el dispositivo de control solar (de índice de transmitancia visible 0.24 ), los valores promedio siguen siendo $\geq 500 \quad$ [lux]. Por otro lado, la iluminación natural disponible $(D A v)$ y área iluminada, como se puede observa en la tabla 5, presentan un comportamiento opuesto en ambos espacios. Es decir, mientras que en (A2), la (DAv) disminuye un $2,6 \%$ y el área iluminada un $25 \%-$ alcanzando el $0 \%$-; en (A3), la (DAv) aumenta un $11,7 \%$ y mantienen el área iluminada en $80 \%$.

En relación a la predicción del comportamiento humano y el uso de cortinas como sistema de control solar, podemos observar un mayor porcentaje de horas de uso de este dispositivo de control solar en el espacio (A3) que en el espacio (A2). En (A3) el porcentaje de horas anuales en que se emplea el sistema de control solar (Uso de DCS) - para evitar situaciones de falta de confort en cualquier punto de la grilla - es del $70 \%$. En el caso de (A2) este porcentaje de horas (Uso de $D C S$ ) se reduce al $21 \%$, esto se debe como fue mencionado, que su fachada - (E) - recibe radiación solar directa tan solo en las horas de la mañana posteriormente el recurso se limita a la radiación solar difusa (Figuras 10 y 11). Es decir, el aula (A3) necesita de un comportamiento más activo de sus usuarios en relación a la manipulación del dispositivos de control solar, que en (A2). Sin embargo, de cumplir con la adecuada utilización de este dispositivos, el espacio (A3) tendrá un $39 \%$ más de autonomía de la iluminación natural (DA500lux) que (A2); reduciendo considerablemente el consumo eléctrico anual por iluminación artificial complementaria.

Figura 9 - Descripción del material RADIANCE generado (.rad). Imágenes del material y temperatura de color correlacionada (TCC) del material $\left(5866^{\circ} \mathrm{K}\right)$

\begin{tabular}{ll}
\hline Vista del Material & Características del Dispositivo de Control Solar (DCS) seleccionado \\
\hline & \# Glazings \\
& \# tropical beige \\
& \# visual transmittance: $4 \%$ \\
& \# visual transmissivity: $26 \%$ \\
& Void glass tbeige \\
0 & 0 \\
& 30.240 .240 .24 \\
\hline
\end{tabular}

Tabla 4 - Resultados obtenidos en los espacios (A2) y (A3) "sin dispositivos de control solar" (Sin DCS) y "con dispositivos de control solar" (Con DCS) en métricas dinámicas y consumos eléctricos del sistema de iluminación artificial complementario (Fd)

\begin{tabular}{cccccccc}
\hline & & \multicolumn{7}{c}{ Métricas Dinámicas [\%] } \\
& DA500lux & DAcon500lux & DAv & Uso de DCS & Área Iluminada & Consumo [KWh] \\
\cline { 1 - 2 } A2 & Sin DCS & 34 & 62 & 14,1 & - & 25 & 373,1 \\
\cline { 2 - 3 } & Con DCS & 13 & 41 & 11,5 & 21 & 0 & 442,1 \\
\multirow{2}{*}{ A3 } & Sin DCS & 57 & 75 & 42,3 & - & 80 & 306,5 \\
& Con DCS & 54 & 74 & 54,0 & 70 & 80 & 300,0 \\
\hline
\end{tabular}


Al profundizar en los datos, podemos destacar que el consumo eléctrico anual de (A2) aumenta 69 [KWh] al emplear dispositivos de control solar (Con DCS), mientras que (A3) disminuye 6.5 [KWh]. Es decir, mientras que (A2) aumenta sus consumos en pos del bienestar de sus usuarios (es decir, neutralizar el $2 \%$ de probabilidad de deslumbramiento), (A3) reducen sus consumos eléctricos con el uso de dispositivos de control (neutralizando el $78 \%$ de probabilidad de deslumbramiento) (Figura 8). Asimismo al comparar ambos espacio, (A3) presenta un ahorro en el consumo eléctrico por iluminación artificial de 142.1 [KWh] en relación a (A2) (Figuras 12 y 13).

\section{Conclusiones}

En el desarrollo del trabajo se han analizado comparativamente diferentes acondicionamientos lumínicos, correspondientes a dos tipologías de aulas representativas del parque edilicio educativo tradicional del sector público de la región. En este análisis fue combinado el uso de espacios reales con modelos predictivos de precisión simulaciones. La importancia de esto radica en un mejor entendimiento de la problemática abordada. Es decir, a partir de las referencias otorgadas por mediciones y relevamientos in situ de los espacios reales, es posible ingresar y calibrar con mayor precisión las variables de input de los simuladores. En el caso de las métricas dinámicas estos input corresponden especificamente a la geometría del espacio, materiales y bases climáticas (MONTEOLIVA; VILLALBA; PATTINI, 2012). Asimismo, de cumplirse con el adecuado uso de los input, estas herramientas predictivas otorgan una mayor representatividad del comportamiento de la iluminación natural en el interior de un espacio determinado. De nor ser así, la evaluación predictiva generaría datos no representativos e interpretaciones erróneas para posibles intervención en el diseño de estrategias de iluminación natural.

Los resultados posibilitaron verificar, en tipologías de aulas con aventanamiento bilateral, la importancia del factor orientación (N-S) en la región y la adecuada relación entre la superficie vidriada y el área del piso - establecido por la normativa nacional vigente IRAM AADL J20 02 (INSTITUTO..., 1970). Esto se evidencia en el espacio (A3) - con/sin dispositivo de control - y en las métricas de autonomía (DA500lux) y autonomía ponderada (DAcon500lux) de iluminación natural; obteniendo valores por encima del $54 \%(=55.5 \%)$ y $74 \%(=74.5 \%)$, respectivamente. Sin embargo, esta tipología tradicional presenta ocasionalmente similitudes a un "sistema de redirección". Es decir, sin ser planificado con este sentido, el techo correspondiente al pasillo exterior $(\mathrm{N})$ y su recubrimiento con un material semi-especular (membrana alfáltica) de alta reflectancia en el espectro visible >80 [\%], generan una estrategía bioclimática.

Figura 10 - Uso predictivo de los dispositivos de control solar (textil tropical) en el espacios (A2)

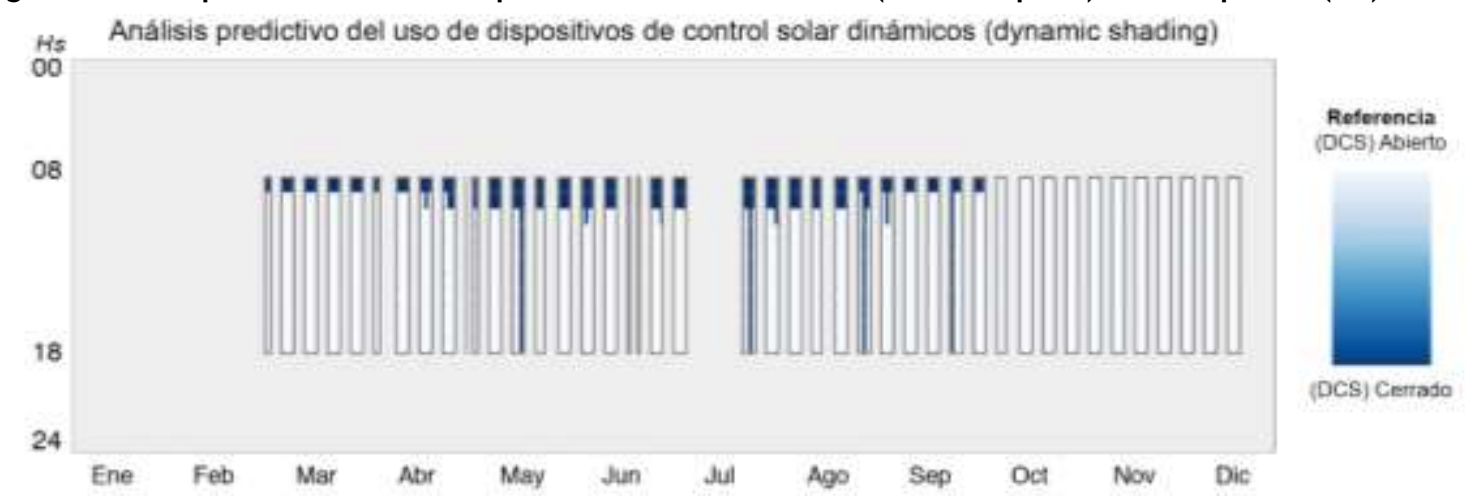

54 Monteoliva, J. M.; Villalba, A.; Pattini, A. E. 
Figura 11 - Uso predictivo de los dispositivos de control solar (textil tropical) en el espacios (A3)

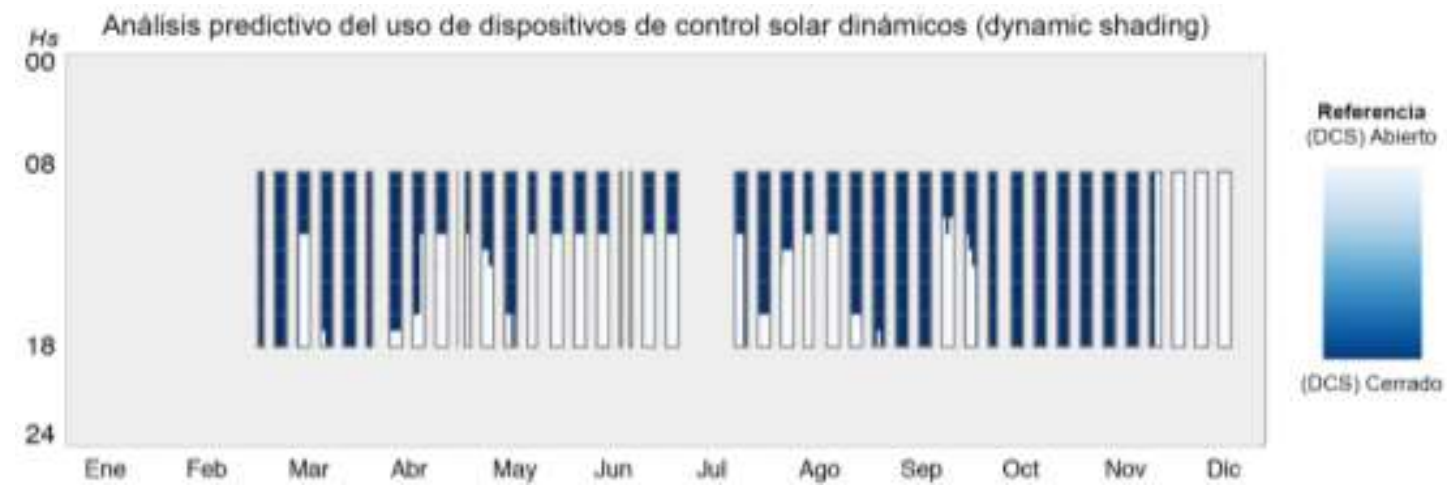

Figura 12 - Uso predictivo del sistema de iluminación artificial complementario en el espacio (A2) con el uso de dispositivos de control solar (con DCS)

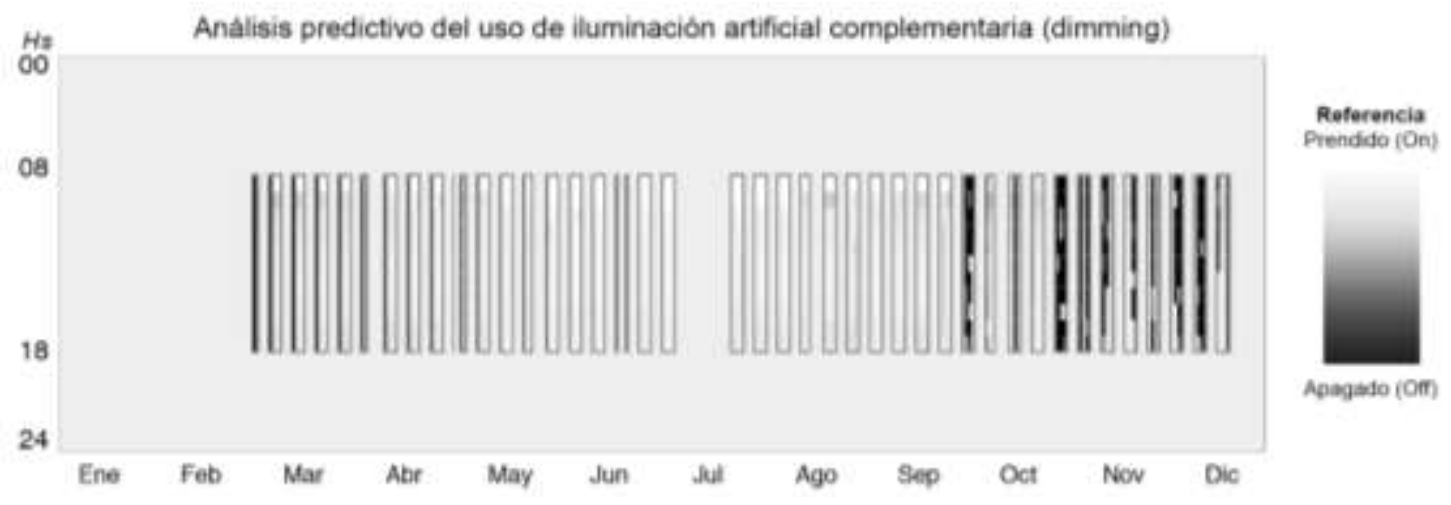

Figura 13 - Uso predictivo del sistema de iluminación artificial complementario en el espacio (A3) con el uso de dispositivos de control solar (con DCS)

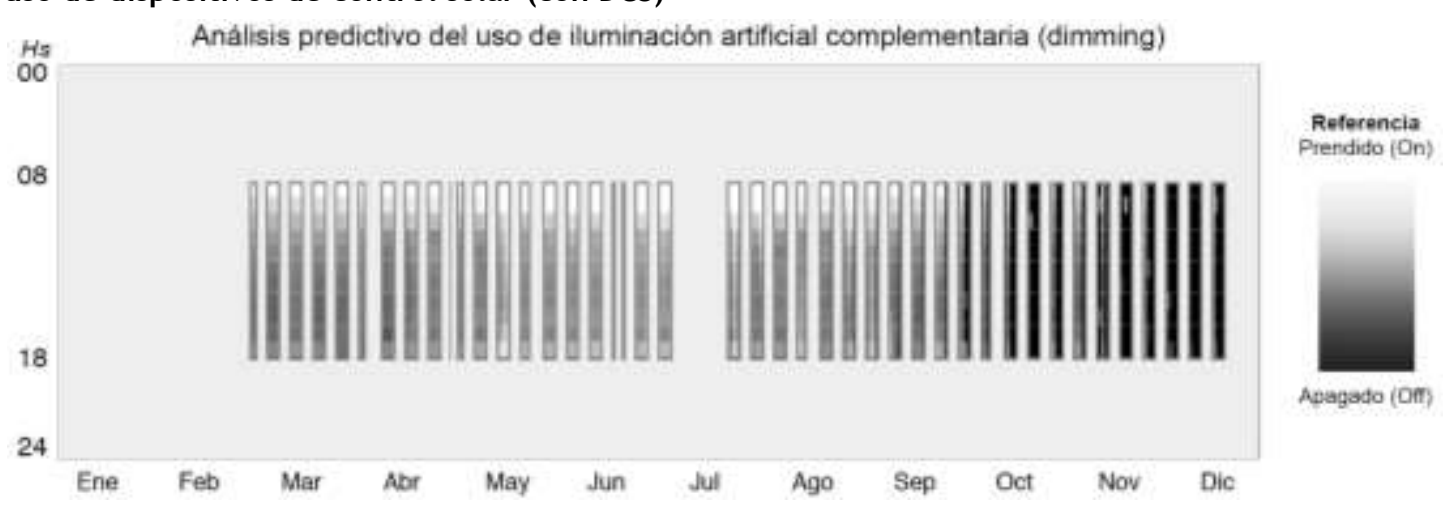

Por otra parte, con el objetivo de incorporar un rango útil más ajustable al estudio de "aulas", fue propuesta como métrica complementaria a iluminación natural autónoma (DA500lux) y ponderada (DAcon500lux), la iluminación natural disponible $(D A v)$. Es decir, el rango útil tienen sus comienzos con el índice de iluminación natural útil (UDI) (REINHART; MARDALJEVIC y ROGERS, 2006). Éste presenta, tres rangos: $<100$ [lux]; 100-2000 [lux] o "rango útil" y > 2000 [lux]. Como se puede observar el rango útil venia expresado por defecto entre los 100-2000 [lux]; aspecto que limita la posibilidad de ajustar los criterios de iluminancia mínimas requeridas, por ejemplo en este estudio de 500 [lux]. A partir del uso de $(D A v)$ el valor límite inferior del rango es ajustables y posibilita profundizar en el estudio del acondicionamiento lumínico de los espacios. En nuestro estudio en particular, permite no sólo, establecer que la autonomía y autonomía ponderada del espacio (A3) es del $55.5 \%$ promedio, sino también que el $7.35 \% \quad(55.5 \%$ (DA500lux) - 48.15\% (DAv)) de la iluminación natural que ingresa al local, se encuentran por 
encima de los 2000 [lux] - valores generadores de posibles situaciones de deslumbramiento. Asimismo cabe mencionar, la importancia de trabajar con rangos ajustados a las regiones soleadas. Por esta razón, se encuentran actualmente en desarrollo, experimentos que buscan ajustar los parámetros mínimos y máximos, generados a partir de estudios y criterios propios acordes a la región.

Por otra parte, el estudio permitió cuantificar el impacto del uso de dispositivos de control en relación a dos tipologías de aulas diferentes. En relación a este aspecto cabe resaltar la importancia del análisis del comportamiento de un elemento de control solar en situaciones de uso específico. Esto se debe, como se demuestra en este trabajo, a que un dispositivo de control puede mejorar el factor iluminación natural en un espacio con determinadas características (A3) o desmejorarlo (A2), según la orientación del espacio y las características del entorno. A partir de ello, se plantea en futuros estudios, el análisis del comportamiento lumínico de estas aulas bajo el impacto de otros sistemas de control solar, tales como venecianas o estantes de luz. Cabe destacar, que todos estos análisis fueron contrastados con los consumos eléctricos anuales del sistemas de iluminación artificial complementaria propuestos de fotosensores y dimerizado $(F d)$. La selección de este sistema $(F d)$ radica en dos motivos: (1) las limitaciones mencionadas en el modelo predictivo de Lightswitch (REINHART, 2004) y (2) la necesidad de generar soluciones con tecnologías accesibles a la región que permitan, no sólo regular el uso responsable de los sistemas de iluminación artificial complementarios, sino también acordes al ámbito en estudio.

Otro aspecto a tener en cuenta, como parte de la discusión, son los indicadores establecidos por la normativa nacional vigente (IRAM AADL serie J). Estas normas están basadas en parámetros de iluminancia, distribución y contraste de luminancia para condiciones de iluminación de carácter artificial, cumpliendo con las norma generales de “buena iluminación" (IRAM- AADL J20 02, 20 03 y 20 05). Para las condiciones de iluminación natural, la normativa propone como único índice representativo al coeficiente de luz diurna (CLD o DF). Sin embargo, este índice está determinado para condiciones de cielo nublado, cielo no característico de nuestra región. Es decir, el (CLD), no es un indicador representativo para la mayor parte del territorio nacional donde en regiones como las de Cuyo (Mendoza, San Juan, San Luis y La Rioja. Argentina) el 85\% de los días del año presentan cielo claro con sol.
Por último, como fue mencionado en el trabajo, el paradigma estático o "point-in-time" se basaba en parámetros constantes dados por una fuente artificial de igual características, similares a los establecidos por las normativas nacionales. Sin embargo, la dinámica de la fuente natural planteó y plantea nuevos desafío. El presente trabajo asume una clara posición a favor del uso de metodologías dinámicas, como el análisis predictivo más representativo del factor iluminación natural en el espacio educativo. A partir de este nuevo enfoque, podremos profundizar en la búsqueda de reducciones significativas de los consumos eléctricos (iluminación) en los edificios de uso diurno. Donde no sólo se incorpore nuevas tecnologías de sistemas de iluminación artificial complementaria, sino también, nuevos dispositivos de control solar en pos del bienestar de los usuarios.

\section{Referencias}

\section{BAKER N.; STEEMERS K. Daylight Design of Buildings. London: James \& James, 2002.}

BODART, M.; DE HERDE, A. Global Energy Savings in Office Buildings By Use of Daylighting. Energy and Buildings, v. 34, n. 5, p. 421-429, 2002.

CANTÓN, A.; DE ROSA, C.; KASPERIDUS, H. Sustentabilidad del Bosque Urbano en el Área Metropolitana de la Ciudad de Mendoza: análisis y diagnóstico de la condición de las arboledas. Avances en Energías Renovables y Medio Ambiente, v. 7, n. 1, p. 29-34, 2003.

CÓRICA, L. Comportamiento de la Luz Natural en Entornos Urbanos Representativos del Modelo Oasis en Regiones Áridas. Caso de Estudio: ciudad de Mendoza. Tucumán, 2009. Tesis (Doctorado) - Departamento de Luminotecnia, Luz y Visión Herberto Büller, Universidad Nacional de Tucumán, . Tucumán, 2009.

DIRECCIÓN GENERAL DE ESCUELAS. Calendario Escolar Ciclo Lectivo. Gobierno de Mendoza, 2012. Disponible en:

<http://bases.mendoza.edu.ar/aplicaciones/legales/ gestion/documentos/ffd503_2616.pdf>. Acceso en: 08 jul. 2014.

DUNN, R. et al. Light Up Their Lives: a research on the effects of lighting on children's achievement and behavior. The Reading Teacher, v. 38, n. 19, p. 863-869, 1985.

EVANS, G. W.; FERGUSON, K. T. Built Environment and Mental Health. In: NRIAGU, J. O. (Ed.). Encyclopedia of Environmental

Health. Burlington: Elsevier, 2011. p. 446-449. 
FONTOYNONT, M. Daylight Performance of Buildings. Lyon: ENTPE, 1999.

HESCHONG, L. Daylighting in Schools: an investigation into the relationship between daylighting and human performance. Fair Oaks: Heschong Mahone Group to Pacific Gas and Electric, 1999.

\section{INSTITUTO ARGENTINO DE}

NORMALIZACIÓN Y CERTIFICACIÓN. Asociación Argentina de Luminotecnia. J20-04: iluminación en escuelas: características. Bahia Blanca, 1974.

\section{INSTITUTO ARGENTINO DE}

NORMALIZACIÓN Y CERTIFICACIÓN. J2002: iluminación natural en edificios: condiciones generales y requisitos especiales. Bahia Blanca, 1970.

JAKUBIEC, J. A.; REINHART, C. F. The 'Adaptive Zone': a concept for assessing glare throughout daylit spaces. Lighting Research \& Technology, v. 44, n. 2, p. 149-170, oct. 2011.

LI, D. H. W., LAU C. S.; LAM, J. C. Predicting Daylight Illuminance by Computer Simulation Techniques. International Journal of Lighting Research and Technology, 36, n. 2, p. 113-129, may. 2004.

MCNEEL, R. et al. Rhinoceros Version 4.0. 2010. Service Release.

MINISTERIO DE EDUCACIÓN DE LA REPÚBLICA ARGENTINA. Dirección de Infraestructura. Criterios y Normativa Básicas de Arquitectura Escolar. Oct. 1998.

MONTEOLIVA, J. M. Influencia del Diseño de la Iluminación en Aulas Sobre la Eficacia Atencional en Niños: impacto de la luz natural. Mendoza: CONICET, 2010. Beca Interna Tipo II.

MONTEOLIVA, J. M.; PATTINI, A. Iluminación Natural en Aulas: análisis predictivo dinámico del rendimiento lumínico-energético en clima soleados. Ambiente Construído, Porto Alegre, v. 13, n. 4, p. 235-248, jul./set. 2013.

MONTEOLIVA, J. M.; VILLALBA, A.; PATTINI, A. Impacto de la Utilización de Bases Climáticas Regionales en la Simulación de Alta Precisión de Iluminación Natural. Avances en Energías Renovables y Medio Ambiente, v. 16, 2012.

OKURA, S.; HESCHONG, L.; WRIGHT, R. Skylighting and Retail Sales. ACEEE Summer Study on Energy Efficiency in Buildings, v. 8, p. 245-56, 2000.

PATTINI, A. La Luz Natural en las Escuelas: aprovechamiento y control de la luz solar en aulas. Buenos Aires: Dunken, 2009.
PEREZ, R. et al. Modeling Daylight Availability and Irradiance Components From Direct and Global Irradiance. Solar Energy, v. 44, n. 5, p. 271-289, 1990.

PEREZ, R.; SEALS, R.; MICHALSKY, J. AllWeather Model for Sky Luminance Distribution: preliminary configuration and validation. Solar Energy, v. 50, n. 3, p. 235-245, 1993.

PHILLIPS, R. W. Educational Facility Age and the Academic Achievement of Upper Elementary School Students. Georgia, 1997. Thesis (Doctoral Dissertation) - University of Georgia, Georgia, 1997.

RAMOS, G.; GHISI, E. Analysis of Daylight Calculated Using the EnergyPlus Program. Renewable and Sustainable Energy Reviews, v. 14, n. 7, p. 1948-58, 2010.

REINHART, C. F. Tutorial on the Use of DAYSIM Simulations for Sustainable Design. Ottawa: Institute for Research in Construction, National Research Council Canada, 2006.

REINHART, C.; MARDALJEVIC, J.; ROGERS, Z. Dynamic Daylight Performance Metrics for Sustainable Building Design. LEUKOS, v. 3, n. 1, p. 1-20, 2006.

REINHART, C. F. et al. DIVA For Rhino Version 2.0. 2011. Disponíble en: <http://www.diva-forrhino.com/>. Acceso em: 25 nov. 2013.

REINHART, C. F. LIGHTSWITCH 2002: a model for manual and automated control of electric lighting and blinds. Solar Energy, v. 77, n. 1, p. 15-28, 2004.

ROGERS, Z. Daylighting Metric Development Using Daylight Autonomy Calculations. Sensor Placement Optimization Tool. Boulder, Colorado, USA: Architectural Energy Corporation, 2006.

TAYLOR, A.; GOUSIE, G. The Ecology of Learning Environments For Children. CEFPI Journal, v. 26, n. 4, p. 23-28, 1988.

VERSAGE, R.; MELO, A. P.; LAMBERTS, R. Impact of Different Daylighting Simulation Results on the Prediction of Total Energy Consumption. In: NATIONAL CONFERENCE OF IBPSA-USA, 5., New York, 2010. Proceedings... New York, 2010.

VILLALBA, A. M.; PATTINI, A. E.; CÓRICA, M. L. Análisis de las características morfológicas de las envolventes edilicias y del entorno urbano desde la perspectiva de la iluminación natural. Ambiente Construído, Porto Alegre, v. 12, n. 4, p. 159-175, out./dez. 2012. 
WARD, G.; SHAKESPEARE, R. A. Rendering with Radiance. San Francisco: Morgan Kaufmann Publishers, 1998.

WIENOLD, J.; CHRISTOFFERSEN, J.

Evaluation Methods and Development of a New

Glare Prediction Model for Daylight Environments With the Use of CCD Cameras. Energy and

Buildings, v. 38, n. 7, p. 743-757, 2006.

WIENOLD, J.; CHRISTOFFERSEN, J. Towards a New Daylight Glare Rating. Berlin:

LuxEuropa, 2005.

WIENOLD, J. Evalglare: a new RADIANCE: based tool to evaluate glare in office spaces. In: INTERNATIOL RADIANCE WORKSHOP, 3 , Fribourg, 2004. Proceedings... Fribourg, 2004.

WU, W.; NG, E. A Review of the Development of Daylighting in Schools. Lighting Research and Technology, v. 35, n. 2, p. 111-125, 2003.
YANNAS, S. Design of Educational Buildings. London: E. G. Bond Ltd., 1994. Environment \& Energy Studies Programme. Architectural Association Graduate School. European Commission, Directorate General XII for Science Research and Development

\section{Este trabajo fue financiado con los proyectos}

- PICTO ENARGAS 2009-0133: Desarrollo y Estudio de Comportamiento Energético de Precisión de Carpinterías Exteriores y Elementos de Control Solar de Bajo Costo. FONCYT, Agencia Nacional de Promoción Científica y Tecnológica, Argentina; y

- PIP 0133 CONICET: Barreras que Obstaculizan el Uso De Iluminación Natural en el Hábitat Construido. Identificación y Propuestas de Superación en Climas Soleados.

Juan Manuel Monteoliva

Laboratorio de Ambiente Humano y Vivienda - INCIHUSA | Consejo Nacional de Investigaciones Científicas y Técnicas | Avenida Ruiz Leal s/n, Pque Gral San Martín | Mendoza - Argentina | CP 5500 | Tel.: 54 (261) 524-4322 | E-mail: jmonteoliva@mendoza-conicet.gob.ar

Ayelén Villalba

Laboratorio de Ambiente Humano y Vivienda - INCIHUSA | Consejo Nacional de Investigaciones Científicas y Técnicas | E-mail: avillalba@mendoza-conicet.gob.ar

\section{Andrea Elvira Pattini}

Laboratorio de Ambiente Humano y Vivienda - INCIHUSA | Consejo Nacional de Investigaciones Científicas y Técnicas | Tel.: 54 (261) 524 4345 | E-mail: apattini@mendoza-conicet.gob.ar

Revista Ambiente Construído

Associação Nacional de Tecnologia do Ambiente Construído

Av. Osvaldo Aranha, $99-3^{\circ}$ andar, Centro

Porto Alegre - RS - Brasil

CEP 90035-190

Telefone: +55 (51) 3308-4084

Fax: +55 (51) 3308-4054

www.seer.ufrgs.br/ambienteconstruido

E-mail: ambienteconstruido@ufrgs.br 